\title{
Fixed points for one-dimensional particle system with strong interaction
}

\author{
V. A. Malyshev
}

\begin{abstract}
We consider hamiltonian $N$ particle system on the finite segment with nearest-neighbor Coulomb interaction and external force $F$. We study the fixed points of such system and show that the distances between neighbors are asymptotically, for large $N$, the same for any $F$.
\end{abstract}

\section{Introduction}

The main goal of the paper is to present a peculiar equilibrium model of large system of interacting particles with strong interaction. We study properties of this system for zero temperature, that is we study ground states of the model. Moreover, it exhibits phenomena somewhat unusual for the modern mathematical statistical physics. Namely, the local equilibrium (defining the global equilibrium) in this model lives on the scale much smaller than normal microscale, see more discussion at the final remarks.

We consider the system of $N$ classical identical point particles on the interval $[0, L] \in R$

$$
0 \leq x_{1}(t)<\ldots<x_{N}(t) \leq L
$$

The dynamics is defined by the system of $N$ equations

$$
m \frac{d^{2} x_{i}}{d t^{2}}=-\frac{\partial U}{\partial x_{i}}+F\left(x_{i}\right)-A \frac{d x_{i}}{d t}
$$

where the interaction is

$$
U\left(x_{1}, \ldots, x_{N}\right)=\sum_{i=2}^{N} V\left(x_{i}-x_{i-1}\right)
$$

It is assumed that the potential $V(x)=V(-x)>0$ is repulsive, that is

$$
f(r)=-\frac{d V(r)}{d r}>0, r=|x|
$$

It is also assumed that $f$ is sufficiently smooth, monotone decreasing and

$$
f(r) \sim_{r \rightarrow 0} \alpha r^{-a}, \alpha>0, a>1
$$

The example is

$$
f(r)=\alpha r^{-a}
$$


Further on we take $\alpha=1$.

It is also assumed that the external force $F$ is continuous function on $[0, L]$. We assume completely inelastic boundary conditions, that is when a particle $x_{1}(t)$ reaches point 0 having the velocity $v_{1}(t)<0$ then immediately its velocity $v_{1}(t+0)$ becomes 0 , the particle stays at 0 until the force $F(0)-f\left(x_{2}(t)\right)$ becomes positive. Similarly for the particle $x_{N}(t)$ at the point $L$.

It follows from the elementary theory of ODE that for any initial data

$$
\left(x_{i}(0), v_{i}(0)\right), i=1, \ldots, N, x_{i}(0)<x_{i+1}(0)
$$

the solution exists and is unique for $t \in[0, \infty)$. Moreover, the order of particles cannot change. We will study the fixed points of this system.

For zero external force $F(x) \equiv 0$ the point $\left(x_{1}, \ldots, x_{N}\right)$ with $x_{k}=\frac{L(k-1)}{N-1}$ is evidently the unique fixed point. In fact, the particles $x_{1}, x_{N}$ reaching the boundary stay there forever. Moreover, the force acting on particle $i=2, \ldots, N-1$ is zero only if $x_{i}-x_{i-1}=x_{i+1}-x_{i}$. This is only possible if

$$
x_{i}-x_{i-1}=\frac{L}{N-1}, i=2, \ldots, N
$$

Ground states with different interactions (namely, $V$ has a distinguished minimum and not necessarily nearest neighbors), but without external force, for classical finite and infinite particle systems were intensively studied during last 30 years, see for example [3, 4, , 5, 6, 7, 8]. Main results in these papers concern periodicity of the ground states.

Further on we consider non-zero external force $F(x) \neq 0$, and our main concern is the degree of non-periodicity of the ground states.

\section{Results}

We consider mostly the case when $N$ is sufficiently large. For small number of particles there can be ugly situations. It is easy to construct examples with a continuum of fixed points: let $N=3$ and consider 3 particles on the interval $[0,1]$ at the points $x_{1}=0<x_{2}<x_{3}=1$. Take some $0<y<\frac{1}{2}$ and choose external force as

$$
F(x)=f(1-x)-f(x), x \in[y, 1-y]
$$

and arbitrary otherwise. Then any $x_{2}$ in $[y, 1-y]$ defines the fixed point $\left(0, x_{2}, 1\right)$.

Lemma 1 For any $N \geq 3$ there is not more than one fixed point with $x_{1}=0$ and given $x_{2}$.

Lemma 2 There is $N_{0}>0$ such that for $N>N_{0}$ any fixed point $\left(x_{1}, \ldots, x_{N}\right)$ has $x_{1}=0, x_{N}=$ $L$.

Theorem 1 Assume that $F(x)$ is non-increasing, that is $F(x) \geq F(y)$ if $x<y$. Then the following assertions hold:

- fixed point exists and is unique, denote it $\left(x_{1}, \ldots, x_{N}\right)$;

- for $k=2, \ldots, N$ as $N \rightarrow \infty$ uniformly in $k$

$$
x_{k}-x_{k-1} \sim \frac{L}{N}
$$


- if moreover $F(x)$ is smooth and $A>0$ then for any initial conditions the solution of (1) converges to this fixed point;

- By $\{x \leftrightarrow L-x\}$-symmetry all these statements hold for non-decreasing $F$.

The convergence statement does not concern the main goal of the paper and is given for completeness.

Only the asymptotics (5D) is a bit surprising. The conclusion is that in the strongly interacting particle system, the macroscopic external force is not seen on the common microscopic scale (that is on the scale $\frac{L}{N}$ - the inverse density of particles), but controlled on much finer scale. One can see this scale from the proof of the theorem and, more clearly, is exhibited as the second term of the asymptotic expansion in the next theorem.

Theorem 2 Assume that $f(x)=x^{-a}$, and that the external force is constant, that is $F(x)=F$. Then for $k=1, \ldots, N-1$ as $N \rightarrow \infty$

$$
\left(x_{k+1}-x_{k}\right)-\frac{L}{N-1} \sim \frac{F L^{a}}{a} N^{-a-1}\left(\frac{N}{2}-k\right)
$$

\section{Proofs}

Proof of Lemma 2 Assume $x_{1}>0$. Then the equilibrium condition for the particle $x_{1}$ is

$$
f\left(x_{2}-x_{1}\right)=F\left(x_{1}\right)
$$

It follows that $F\left(x_{1}\right)>0$. By monotonicity of $f$, it follows from (7) that $x_{2}-x_{1}$ is uniquely defined, and does not depend on $N$. We also have the following triangular system - equilibrium conditions for the particles $k=2, \ldots, N-1$

$$
f\left(x_{k+1}-x_{k}\right)=f\left(x_{k}-x_{k-1}\right)+F\left(x_{k}\right)
$$

From (8) we can find by induction $x_{3}-x_{2}, \ldots, x_{N}-x_{N-1}$ in a unique way. $F\left(x_{k}\right)$ can be negative but $f\left(x_{k}-x_{k-1}\right)+F\left(x_{k}\right)$ should be non-negative for all $k$, otherwise such fixed point would not exist. Then for any $k=2, \ldots, N$ we have from (77) and (8)

$$
f\left(x_{k}-x_{k-1}\right)=\sum_{i=1}^{k-1} F\left(x_{i}\right)
$$

This gives, for $C=\sup F$, the inequalities

$$
0 \leq f\left(x_{k}-x_{k-1}\right)<C k \Rightarrow x_{k}-x_{k-1}>\left(\frac{1}{f\left(x_{k}-x_{k-1}\right)}\right)^{\frac{1}{a}}>C k^{-\frac{1}{a}}
$$

If it appears that

$$
x_{1}+\left(x_{2}-x_{1}\right)+\ldots+\left(x_{N}-x_{N-1}\right)=x_{N}=L
$$

then $\left(x_{1}, \ldots, x_{N}\right)$ is a fixed point. If $x_{N}<L$ then

$$
F\left(x_{N}\right)=-f\left(x_{N}-x_{N-1}\right)
$$

and it is only possible if

$$
\sum_{k=1}^{N} F_{k}=0
$$

In any case, $x_{N} \leq L$ is impossible for sufficiently large $N$, as due to $\frac{1}{a}<1$, the series $\sum k^{-a^{-1}}$ diverges. For the point $x_{N}$ the proof is symmetric. 
Proof of Lemma 1 Instead of (17) we have for the point $x_{1}=0$ much weaker condition

$$
f\left(x_{2}\right) \geq F(0)
$$

Let $x_{2}-x_{1}=x_{2}$ be given such that (otherwise fixed point could not exist)

$$
f\left(x_{2}-x_{1}\right)+F\left(x_{2}\right)>0
$$

Then we uniquely find $x_{3}-x_{2}$ from

$$
f\left(x_{3}-x_{2}\right)=f\left(x_{2}-x_{1}\right)+F\left(x_{2}\right)
$$

Proceeding by induction we find uniquely $x_{3}, \ldots, x_{N}$ as functions of $x_{2}$. If

$$
x_{N}<L, f\left(x_{N}-x_{N-1}\right)+F=0
$$

or

$$
x_{N}=L, f\left(x_{N}-x_{N-1}\right)+F>0
$$

then $\left(x_{1}, \ldots, x_{N}\right)$ is the fixed point, otherwise $\left(x_{1}, \ldots, x_{N}\right)$ is not a fixed point. That is $x_{k}, k>2$, are the functions $x_{k}\left(x_{2}\right)$ of $x_{2}$.

Now we will prove theorem 1.

Proof of uniqueness Put

$$
\Delta_{k}=\Delta_{k}^{(1)}=x_{k+1}-x_{k}
$$

We want to prove that the functions $x_{k}\left(x_{2}\right)$, defined in the proof of lemma 1, are strictly increasing. Then there is not more than one value of $x_{2}$ such that $x_{N}=x_{N}\left(x_{2}\right)=L$, that gives uniqueness.

For example, if $x_{2}$ increases then the right-hand side of (11) decreases, thus $f\left(x_{3}-x_{2}\right)$ decreases and $\Delta_{2}=x_{3}-x_{2}$ increases. By induction, if $x_{k}<x_{k}^{\prime}, \Delta_{k-1}<\Delta_{k-1}^{\prime}$ then $F\left(x_{k}^{\prime}\right) \leq$ $F\left(x_{k}\right), f\left(\Delta_{k-1}\right)>f\left(\Delta_{k-1}^{\prime}\right)$. It follows from (8) that $f\left(\Delta_{k}\right)>f\left(\Delta_{k}^{\prime}\right)$. Thus $\Delta_{k}^{\prime}>\Delta_{k}$ and

$$
x_{k+1}^{\prime}=x_{k}^{\prime}+\Delta_{k}^{\prime}>x_{k}+\Delta_{k}=x_{k+1}
$$

We know that the function $x_{N}\left(x_{2}\right)$ is continuous. Thus, to prove existence one should prove that this function does not grow too fast with $N$. In this proof we get also the asymptotics for $\Delta_{k}$.

Existence and asymptotics Note that for all $k$

$$
f\left(x_{k+1}-x_{k}\right)=f\left(x_{2}\right)+\sum_{i=2}^{k} F\left(x_{i}\right)
$$

By (21) for $x \rightarrow 0$

$$
f(x)=\frac{1}{x^{a}}(1+\epsilon(x)), \epsilon(x)=o(1)
$$

We will put for large $N$

$$
x_{2}=\frac{L}{N-1}(1+b), b=b(N)=o(1)
$$


From (12) we get that there exists $C>0$ such that for all $k$

$$
-C k+f\left(\frac{L}{N-1}(1+b)\right)<f\left(x_{k+1}-x_{k}\right)<C k+f\left(\frac{L}{N-1}(1+b)\right)
$$

or

$$
\left(f\left(\frac{L}{N-1}(1+b)\right)+C k\right)^{-\frac{1}{a}}<\Delta_{k}\left(1+\epsilon\left(\Delta_{k}\right)\right)^{-\frac{1}{a}}<\left(f\left(\frac{L}{N-1}(1+b)\right)-C k\right)^{-\frac{1}{a}}
$$

But

$$
\begin{gathered}
\left(f\left(\frac{L}{N-1}(1+b)\right) \pm C k\right)^{-\frac{1}{a}}=\left(\left(\frac{L}{N-1}(1+b)\right)^{-a}\left(1+\epsilon\left(\frac{L}{N-1}(1+b)\right)\right) \pm C k\right)^{-\frac{1}{a}}= \\
=\frac{L}{N-1}\left((1+b)^{-a}\left(1+\epsilon\left(\frac{L}{N-1}(1+b)\right)\right) \pm C k \frac{L^{a}}{(N-1)^{a}}\right)^{-\frac{1}{a}}= \\
=\frac{L}{N-1}\left(1+b-\frac{1}{a} \epsilon\left(\frac{L}{N-1}(1+b)\right) \mp \frac{1}{a} C k \frac{L^{a}}{(N-1)^{a}}+o\left(|b|+|\epsilon|+k \frac{L^{a}}{(N-1)^{a}}\right)\right)
\end{gathered}
$$

From (13) it follows that uniformly in $k$

$$
\Delta_{k}=O\left(\frac{1}{N}\right)
$$

Then $\epsilon\left(\Delta_{k}\right)=o(1)$ also uniformly in $k$, and we have the following upper bound

$$
\begin{gathered}
\sum_{k=1}^{N-1} \Delta_{k}\left(1+\epsilon\left(\Delta_{k}\right)\right)^{-\frac{1}{a}}=\sum_{k=1}^{N-1} \Delta_{k}+o(1)=x_{N}(b)+o(1) \leq \\
\left.\leq L\left(1+b-\frac{1}{a} \epsilon\left(\frac{L}{N-1}(1+b)\right)\right)+\frac{1}{a} C \frac{(N-2)}{2} \frac{L^{a}}{(N-1)^{a}}+o(\ldots)\right)
\end{gathered}
$$

and similar lower bound with minus in front of $C$. It follows that the equation

$$
x_{N}(b)=L
$$

for $N$ sufficienly large has a unique solution $b$ such that $b=o(1)$.

Proof of Theorem 2 Here we get exact expression for the second term for the asymptotic expression of

$$
\Delta_{k}=x_{k+1}-x_{k}=\frac{L}{N-1}\left(1+\delta_{k}\right)
$$

that is the asymptotics for $\delta_{k}$ in case of constant $F$. We have from (12)

$$
f\left(x_{k+1}-x_{k}\right)=f\left(x_{2}\right)+(k-1) F
$$

and instead of (13) we have $\left(b=\delta_{1}\right)$

$$
f\left(\frac{L}{N-1}\left(1+\delta_{k}\right)\right)-f\left(\frac{L}{N-1}(1+b)\right)=(k-1) F
$$

or

$$
\left(1+\delta_{k}\right)^{-a}-(1+b)^{-a}=\left(\frac{L}{N-1}\right)^{a}(k-1) F
$$


We can rewrite

$$
\begin{gathered}
1+\delta_{k}=\left[(1+b)^{-a}+\left(\frac{L}{N-1}\right)^{a}(k-1) F\right]^{-\frac{1}{a}}= \\
=\left[1-a b+O\left(b^{2}\right)+\left(\frac{L}{N-1}\right)^{a}(k-1) F\right]^{-\frac{1}{a}}=1+b-F a^{-1}\left(\frac{L}{N-1}\right)^{a}(k-1)+g(b, N, k)
\end{gathered}
$$

where

$$
g(b, N, k)=O\left(b^{2}+b\left(\frac{L}{N-1}\right)^{a}(k-1)+\left(\frac{L}{N-1}\right)^{2 a}(k-1)^{2}\right)
$$

Make summation over $k=1, \ldots, N-1$, and using condition

$$
\sum \delta_{k}=0
$$

we get

$$
\sum_{k=1}^{N-1} \delta_{k}=0=(N-1) b-F a^{-1}\left(\frac{L}{N-1}\right)^{a} \sum_{k=1}^{N-1}(k-1)+\sum_{k=1}^{N-1} g(b, N, k)
$$

From there

$$
b=(N-1)^{-1} F a^{-1}\left(\frac{L}{N-1}\right)^{a} \frac{(N-1)(N-2)}{2}+G(b, N)
$$

where

$$
G(b, N)=O\left(b^{2}+b\left(\frac{L}{N-1}\right)^{a}(N-1)+\left(\frac{L}{N-1}\right)^{2 a}(N-1)\right)
$$

Thus there is a unique solution for $b$, asymptotically equal to

$$
b \sim \frac{F L^{a}}{2 a} N^{-a+1}
$$

Then from (14) we have

$$
\delta_{k} \sim \frac{F L^{a}}{a} N^{-a}\left(\frac{N}{2}-k\right)
$$

Convergence Here we will prove that if $A>0$ then for any initial conditions and $t \rightarrow \infty$ the solution converges to the fixed point.

Define the potential energy

$$
\left.W\left(x_{1}, \ldots, x_{N}\right)=U\left(x_{1}, \ldots, x_{N}\right)-\sum_{k=1}^{N} \int_{0}^{x_{k}} F(x) d x\right)
$$

Then the equations

$$
\frac{\partial W}{\partial x_{k}}=0
$$

are the fixed point equations for non boundary points. Note first that $W$ cannot have minimum in the following cases: 1) $\left.\left.x_{1}>0, x_{N}<L, 2\right) x_{1}=0, x_{N}<L, 3\right) x_{1}>0, x_{N}=L$. This is because the equations (15), by Lemma 2, do not have solution correspondingly for 1) $k=1, \ldots, N, 2$ ) $k=2, \ldots, N, 3) k=1, \ldots, N-1$.

It follows that minima can be only if $x_{1}=0 ; x_{N}=L$. Moreover, the equations (15) for $k=2, \ldots, N-1$ define both the fixed point and the minimum of $W$. As these equations have unique solution, these points coincide.

The known equality 


$$
\begin{aligned}
\frac{d H}{d t} & =\sum_{i}\left(\frac{\partial H}{\partial p_{i}} \frac{d p_{i}}{d t}+\frac{\partial U}{\partial x_{i}} \frac{d x_{i}}{d t}-F\left(x_{i}\right) \frac{d x_{i}}{d t}\right)=\sum_{i}\left(v_{i} \frac{d p_{i}}{d t}+\left(\frac{\partial U}{\partial x_{i}}-F_{i}\right) v_{i}\right)= \\
& =\sum_{i} v_{i}\left(-\frac{\partial U}{\partial x_{i}}-A v_{i}+F\left(x_{i}\right)\right)+\sum_{i}\left(\frac{\partial U}{\partial x_{i}}-F\left(x_{i}\right)\right) v_{i}=-A \sum v_{i}^{2}
\end{aligned}
$$

shows that the full energy $H=\frac{p^{2}}{2 m}+W$ is non-increasing.

Define metrics in the set $\left\{X=\left(x_{1}, \ldots, x_{N}\right)\right\}$ of configurations

$$
\rho(X, Y)=\sum_{k=1}^{N}\left|x_{k}-y_{k}\right|
$$

We want to show that $H$ reaches any neighborhood of the minimum for finite time.

Lemma 3 For any $\epsilon>0$ there are constants $0<\delta=\delta(\epsilon), \tau=\tau(\epsilon)<1$ such that for any initial conditions outside the neighbourhood $O(\epsilon)$ of the fixed point

$$
\int_{0}^{\tau} \sum_{k=1}^{N} v_{k}^{2}(t) d t>\delta
$$

From this lemma the last assertion of Theorem 1 evidently follows.

Proof of the Lemma. For given $X$ denote $F_{k}=F\left(x_{k-1}, x_{k}, x_{k+1}\right)$ the force acting on the particle $k$ at the point $x_{k}$. Denote

$$
\Phi_{0}=\Phi_{0}(X)=\sum_{k=2}^{N-1}\left|F_{k}\right|, \Phi_{1}\left(X_{9}\right)=\sum_{k=2}^{N-1}\left|\frac{\partial F_{k}}{\partial x_{k}}\right|
$$

and consider the set of configurations

$$
A=A\left(D_{1}, D_{2}\right)=\left\{X: \Phi_{0}<D_{0,}, \Phi_{1}<D_{1}\right\}
$$

Fix some $\epsilon>0$ sufficiently small. Then, by smoothness, there exists constant $0<c_{1}=c_{1}(\epsilon)<$ $\infty$ (depending on $N$ ) such that for any $X \in A \backslash O(\epsilon)$

$$
\Phi_{0}>c_{1}
$$

Take some initial conditions $\left(x_{1}, v_{1}\right), \ldots,\left(x_{N}, v_{N}\right)$ with $X=\left(x_{1}, \ldots, x_{N}\right) \in A \backslash O(\epsilon)$ and let $k$ be such that $F_{k}=F_{k}(X)$ is maximal among other $F_{j}$, then

$$
\left|F_{k}\right|>\frac{c_{1}}{N}
$$

The idea is quite simple: if non of the points $y_{k-1}, y_{k+1}$ does not get sufficient speed, then the force $F_{k}$ will give sufficient speed to the particle $k$. More exactly, for

$$
\delta_{1}=\frac{F_{k}}{2 D_{2}}
$$

we have

$$
\left|F_{k}\left(y_{k-1}, y_{k}, y_{k+1}\right)\right|>\frac{1}{2}\left|F_{k}\left(x_{k-1}, x_{k}, x_{k+1}\right)\right|
$$


for any $y_{i}, i=k-1, k, k+1$ with $\left|x_{i}-y_{i}\right|<\delta_{1}$. Then if none of the three points $y_{k-1}, y_{k}, y_{k+1}$ on the time interval $(0, \tau)$ passes the distance greater than $\delta_{1}$, then $y_{k}$ will pass the distance not smaller than

$$
\delta_{1}=\frac{F_{k} \tau^{2}}{2}
$$

Put then

$$
\tau=\sqrt{\frac{2 \delta_{1}}{F_{k}}}
$$

Then we have the alternative: either

$$
\int_{0}^{\tau}\left|v_{k}\right| d \tau>\delta_{1}
$$

or

$$
\int_{0}^{\tau}\left(\left|v_{k-1}\right|+\left|v_{k+1}\right|\right) d \tau>\delta_{1}
$$

Thus

$$
\int_{0}^{\tau}\left(\left|v_{k-1}\right|+\left|v_{k}\right|+\left|v_{k+1}\right|\right) d t>\delta_{1}
$$

Now one can use the Cauchy inequality

$$
\int_{0}^{\tau} v^{2} d \tau \geq \frac{1}{\tau}\left(\int_{0}^{\tau}|v| d \tau\right)^{2}
$$

We remark that no reasonable convergence rate follows from this proof.

\section{Some remarks}

The appearence of finer scale (we call it submicroscale) with macroeffects is now a rare phenomenon. In many equilibrium models of infinite particle systems local equilibrium conditions are defined on the normal microscale. For example, the corresponding problem (for ground states and for Gibbs low temperature states) in the one-dimensional model of elasticity (the Hooke's law), discussed in [2] where the force, acting only on the particle $x_{N}$, produces discrete laplacians

$$
\Delta_{k}^{(2)}=\delta_{k+1}-\delta_{k}=x_{k+1}-2 x_{k}+x_{k-1}
$$

of the order $\frac{1}{N}$.

\section{References}

[1] V. A. Malyshev. Why current flows: a multiparticle one-dimensional model. Theoretical and Mathematical Physics, 2008, v. 155, No. 2, 766-774.

[2] V. A. Malyshev. One-dimensional mechanical networks and crystals. Moscow Math. Journal, 2006, v. 6, No. 2, pp. 353-358.

[3] W. Ventevogel. On the configuration of a one-dimensional system of interacting particles with minimum potential energy per particle. Physica A, 1978, 92, No. 3-4, pp. 343-361.-

[4] M. Duneau, A. Katz. Structural stability of classical lattices in one-dimension. Annales de l'I.H.P., section A, 1984, 41, No. 3, 269-290. 
[5] Ch. Radin. Existence of ground state configurations. Math. Physics Electronic J., 2004, v. 10 .

[6] Ch. Radin. Crystals and Quasicristals: a lattice gas model. Physics Letters, 1986, 114A, No. 7, 381-383.

[7] C. Gardner, Ch. Radin. The infinite-volume ground state of the Lennard-Jones potential. J. Stat. Phys., 1979, v. 20, No. 6, 719-724.

[8] Ch. Radin, L. Schulman. Periodicity of classical ground states. Phys. Rev. Letters, 1983, 51, No. 8, 621-622. 